\title{
ARTICLE Effects of acute and repeated treatment with methocinnamox, a mu opioid receptor antagonist, on fentanyl self-administration in rhesus monkeys
}

\author{
David R. Maguire ${ }^{1,2}$, Lisa R. Gerak ${ }^{1,2}$, Jesus J. Sanchez ${ }^{3}$, Martin A. Javors ${ }^{1,2,3}$, Alex Disney ${ }^{4}$, Stephen M. Husbands ${ }^{4}$ and \\ Charles P. France ${ }^{1,2,3}$
}

\begin{abstract}
Methocinnamox (MCAM), a mu opioid receptor antagonist with a long duration of action, attenuates heroin self-administration in rhesus monkeys, suggesting it could be an effective treatment for opioid use disorder (OUD). This study examined effects of acute and repeated MCAM administration on self-administration of the high-efficacy mu opioid receptor agonist fentanyl and characterized MCAM pharmacokinetics. Four rhesus monkeys self-administered i.v. infusions of fentanyl ( $0.00032 \mathrm{mg} / \mathrm{kg} / \mathrm{infusion})$ or cocaine $(0.032 \mathrm{mg} / \mathrm{kg} / \mathrm{infusion})$. MCAM $(0.1-0.32 \mathrm{mg} / \mathrm{kg})$ or the opioid receptor antagonist naltrexone $(0.001-0.032 \mathrm{mg} / \mathrm{kg}) \mathrm{was}$ injected prior to test sessions to evaluate acute effects. On a separate occasion, $0.32 \mathrm{mg} / \mathrm{kg}$ MCAM was injected every 12 days for 5 total injections to evaluate the effectiveness of repeated treatment. Following acute injection, MCAM and naltrexone decreased fentanyl self-administration on the day of treatment, with attenuation lasting for up to 2 weeks after the larger MCAM dose and $<1$ day after naltrexone. Repeated MCAM administration decreased fentanyl self-administration for more than 2 months without altering cocaine self-administration. MCAM plasma concentrations peaked 15-45 min after injection, with a half-life ranging from 13.7 to $199.8 \mathrm{~min}$, and decreased markedly 1 day after injection. MCAM selectively reduced opioid self-administration and remained effective with repeated administration. Moreover, MCAM was effective at times when plasma levels were very low, suggesting that pharmacodynamic (i.e., pseudoirreversible binding to $m u$ opioid receptors) and not pharmacokinetic factors play a significant role in its long-lasting effects. Taken together with previous studies, these data indicate that MCAM could be a safe, effective, and longacting treatment for OUD.
\end{abstract}

Neuropsychopharmacology (2020) 45:1986-1993; https://doi.org/10.1038/s41386-020-0698-8

\section{INTRODUCTION}

The opioid crisis continues despite the availability of medications that are effective in some patients [1]. The most common evidence-based treatment for opioid use disorder (OUD) includes medication-assisted therapy; currently approved medications comprise the full $m u$ opioid receptor agonist methadone, the partial agonist buprenorphine, and the antagonist naltrexone. While these medications are effective, each has limitations. For example, although agonists such as methadone and buprenorphine replace abused opioids and mitigate risks associated with illicit drug use, treatment with mu opioid receptor agonists promotes physical dependence, carries significant potential for diversion, and increases the risk of dangerous interactions with other drugs such as alcohol and benzodiazepines [e.g., 2, 3]. $\mathrm{Mu}$ opioid receptor antagonists such as naltrexone avoid many of adverse effects associated with agonists; however, naltrexone binds to opioid receptors in a competitive, reversible manner. Therefore, antagonist effects can be surmounted by taking large doses of an agonist. Moreover, naltrexone has a very short duration of action, requiring daily administration (e.g., Revia'), which can result in low treatment adherence. Although an extended release formulation of naltrexone (Vivitrol ${ }^{\oplus}$ ) is available, the surmountability limitation remains, and low treatment adherence and limited effectiveness continue to be significant challenges $[4,5]$. The severity of the opioid crisis taken together with limitations of currently available treatments underscores the urgent need to discover and develop additional safe and effective treatments for OUD [6].

Methocinnamox (MCAM) is an opioid receptor antagonist that binds to but does not dissociate from the mu opioid receptor, rendering the receptor unavailable to other ligands. Binding to $m u$ opioid receptors in this manner suggests an irreversible interaction between MCAM and the receptor; however, unlike prototypical irreversible ligands such as $\beta$-funaltrexamine [7], MCAM does not form a covalent bond with the receptor and is, therefore, considered to be pseudoirreversible. Consistent with its pseudoirreversible antagonist properties, MCAM attenuates effects of $m u$ opioid receptor agonists such as morphine in rodents, with effects persisting for days or weeks following a single administration [8-10]. MCAM also blocks the reinforcing and ventilatory-depressant effects of heroin and reduces choice of the mu opioid receptor agonist remifentanil over food in

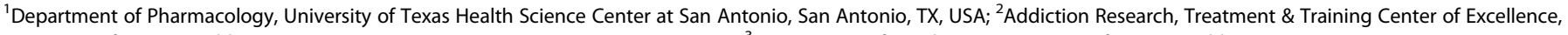

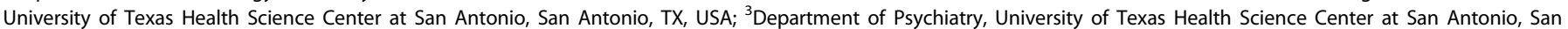
Antonio, TX, USA and ${ }^{4}$ Department of Pharmacy and Pharmacology, University of Bath, Bath, UK

Correspondence: Charles P. France (france@uthscsa.edu)

Received: 13 March 2020 Revised: 13 April 2020 Accepted: 27 April 2020

Published online: 6 May 2020 
rhesus monkeys [11, 12]. Effects of MCAM in monkeys also were remarkably long lasting, with a single injection of MCAM blocking effects of heroin for up to 2 weeks. Moreover, MCAM was effective at doses that did not decrease responding for food or alter physiological parameters such as heart rate, blood pressure, and body temperature [12]. Because MCAM has no pharmacological efficacy at mu opioid receptors, it would be expected to retain many benefits of naltrexone for treating OUD (e.g., no abuse potential, ventilatory depression, or adverse interactions with drugs that decrease ventilation) with the added benefit of being long acting and insurmountable as long as enough MCAM is given to occupy a sufficiently large number of $m u$ opioid receptors. Long-term, insurmountable blockade of $\mathrm{mu}$ opioid receptors would have the advantage of increasing treatment compliance by reducing the frequency of medication administration and mitigating overdose risk insofar as the antagonist effects of MCAM could not be overcome by taking large doses of opioids.

The current study extends this work by evaluating MCAM for its capacity to attenuate the reinforcing effects of the potent and high-efficacy mu opioid receptor agonist fentanyl, which has contributed to a dramatic increase in mortality associated with opioid abuse [13]. Although previous studies demonstrated that MCAM selectively reduces opioid self-administration, it is important to know whether effects of MCAM are sustained with repeated treatment and whether extended treatment with MCAM results in long-term changes that might have important consequences. This study had the following three primary goals: (1) characterize effects (duration and selectivity) of acute treatment with MCAM on fentanyl self-administration; (2) evaluate effectiveness of repeated treatment with MCAM on fentanyl self-administration; and (3) examine MCAM in plasma in order to relate its pharmacokinetic profile to its behavioral effects.

Monkeys were allowed to self-administer fentanyl, and, occasionally, cocaine was substituted for fentanyl to test for the selectivity of antagonism and to determine whether antagonism by MCAM was the result of blockade of mu opioid receptors or some other nonselective effect, such as a generalized reduction in operant behavior, which is an important consideration for evaluating candidate medications for substance use disorders [14]. Moreover, given that MCAM was expected to decrease responding for fentanyl for an extended duration, responding for cocaine would confirm that the i.v. catheter was patent and the monkey would continue working for an i.v. infusion of drug. In addition, effects of MCAM were compared with those of naltrexone, the only mu opioid receptor antagonist currently approved in the US to treat OUD. It was hypothesized that both MCAM and naltrexone would attenuate fentanyl, but not cocaine, self-administration, and that antagonism by MCAM would be much longer lasting than antagonism by naltrexone, consistent with the persistent effects of MCAM at $m u$ opioid receptors in previous studies [11, 12]. Given that long-term blockade of mu opioid receptors might perturb the endogenous opioid system, effects of naltrexone were redetermined periodically throughout the study to assess potential changes in effects (e.g., potency) of drugs acting at opioid receptors.

\section{MATERIALS AND METHODS}

\section{Subjects}

Eight adult rhesus monkeys, 7 males and 1 female, were housed individually in stainless steel cages in colony rooms maintained under a 14/10-h light/dark cycle. Chow (High Protein Monkey Diet; Harlan Teklad, Madison, WI, USA), fresh fruit, peanuts, and treats were provided daily in amounts that maintained healthy sex- and age-appropriate weights, and water was continuously available in the home cage. Experiments were conducted in accordance with guidelines set forth by the Guide for the Care and Use of Laboratory Animals (8th edition; 2011) and protocols were approved by the University of Texas Health Science Center at San Antonio Institutional Animal Care and Use Committee.

\section{Surgery}

Catheters were placed in monkeys participating in the selfadministration experiment. After monkeys were sedated $(10 \mathrm{mg} /$ $\mathrm{kg}$ ketamine), anesthesia was maintained under $2 \mathrm{l} / \mathrm{min}$ oxygen and isoflurane. A 5-fr polyurethane catheter (Access Technologies, Skokie, IL, USA) was inserted and secured into a vein (e.g., jugular or femoral), tunneled subcutaneously (s.c.) to the mid-scapular region, and attached to a s.c. access port (Access Technologies). Meloxicam and penicillin B\&G were given postoperatively; ports and catheters were flushed daily with heparinized sterile saline to maintain catheter patency.

\section{Apparatus}

During sessions, monkeys were seated in chairs (Primate Products Inc., Immokalee, FL, USA). Monkeys participating in the selfadministration experiment were positioned in light- and soundattenuating chambers facing stainless-steel instrument panels with two horizontally aligned levers arranged below translucent disks that could be illuminated green or red. Infusions were delivered intravenously (i.v.) through the s.c. access port via a Huber point needle connected by a catheter extension to a syringe located in a syringe pump (Med Associates, St. Albans, VT, USA) $[12,15]$.

\section{Drug self-administration procedure}

Four monkeys (three males and one female) participated in the self-administration experiment; sessions were conducted once daily $[12,15]$. After monkeys were seated in chairs, weighed, and positioned in operant condition chambers, their ports and catheters were flushed with sterile $0.9 \%$ saline solution. Syringes and extension sets containing the solution available for selfadministration were connected to the Huber needle, and pumps were activated for $15 \mathrm{~s}$ to fill the port and catheter with that solution. The session began with illumination of a red light over one lever (active) for $5 \mathrm{~s}$; a noncontingent infusion of the unit dose of drug available for self-administration in that session was delivered. Thereafter, the green light over the active lever was illuminated, signaling a response period during which 30 consecutive responses on the active lever turned off the green light, turned on the red light for $5 \mathrm{~s}$, turned on the syringe pump until the unit dose of drug was delivered, and initiated a 180-s timeout. During timeouts, all lights were off and responding had no programmed consequence. At the end of the timeout, the green light over the active lever was illuminated, signaling another response period. Responses on the other lever (inactive) reset the response counter on the active lever but otherwise had no scheduled consequence. Sessions ended after $90 \mathrm{~min}$, inclusive of response periods and timeouts.

Effects of acute naltrexone administration Effects of naltrexone were determined while $0.00032 \mathrm{mg} / \mathrm{kg} /$ infusion fentanyl was available for self-administration; this unit dose of fentanyl is at or near the peak of the dose-effect curve for self-administration [e.g., 16, 17] and maintains levels of responding comparable to the unit dose of heroin used in the previous study with MCAM [12]. Monkeys received a s.c. injection $15 \mathrm{~min}$ prior to each session, with saline given prior to baseline sessions and naltrexone given prior to test sessions. Initially, baseline sessions occurred until the number of infusions obtained in each of 3 consecutive sessions did not vary by more than $\pm 20 \%$ of the mean of those sessions; a dose of naltrexone was injected prior to the following (test) session. Thereafter, baseline sessions continued until the number of infusions obtained for a single session 


\section{8}

was within $20 \%$ of the initial baseline mean, at which point another test session occurred [12]. Baseline and test sessions alternated in this fashion until a naltrexone dose-effect curve was established. Naltrexone dose-effect curves were determined on three separate occasions, once prior to each test with MCAM. For the first determination of the naltrexone dose-effect curve, all monkeys received doses of $0.0032,0.01$, and $0.032 \mathrm{mg} / \mathrm{kg}$. Doses of naltrexone in this range attenuate the reinforcing and ventilatory-depressant effects of heroin and other mu opioid receptor agonists in rhesus monkeys [e.g., 11, 12]. For the second and third determinations, doses varied across monkeys, ranging from the largest dose that decreased infusions by less than $20 \%$ of the baseline to the smallest dose that decreased the number of infusions to fewer than 9. For each determination, the number of infusions obtained in baseline sessions that preceded a test were averaged, and that mean value served as the baseline for that particular determination.

\section{Effects of acute MCAM administration}

Effects of acute administration of $0.32 \mathrm{mg} / \mathrm{kg}$ MCAM were established after the first naltrexone dose-effect curve was determined. Prior to the test, $0.032 \mathrm{mg} / \mathrm{kg} /$ infusion cocaine was available for self-administration for at least one session to confirm that cocaine maintained levels of responding above saline in each monkey; this unit dose is at or near the peak of the cocaine self-administration dose-effect curve [e.g., 18, 19], maintains a similar level of responding as the unit dose of fentanyl, and was the same dose used in the previous study with MCAM [12]. Thereafter, $0.00032 \mathrm{mg} / \mathrm{kg} /$ infusion fentanyl was available until the number of infusions obtained in 1 session was within $20 \%$ of the baseline mean established prior to determination of the first naltrexone dose-effect curve. MCAM was given s.c. $60 \mathrm{~min}$ prior to the next session; the doses and pretreatment time were based on published data [12]. Saline $(0.5 \mathrm{ml})$ was injected s.c. $60 \mathrm{~min}$ prior to the sessions conducted on the days immediately preceding and following the MCAM test session. Fentanyl was available during the session conducted on the day of the MCAM injection (Day 0 ) and for 2 days thereafter (Days 1 and 2), then cocaine was available for a single session (Day 3); fentanyl was available again for 3 sessions (Days 4-6) followed by a single session in which cocaine was available (Day 7). This pattern continued, with cocaine substituted for fentanyl every fourth session, for at least 42 sessions (range: 42-46) after MCAM administration. At that point, cocaine sessions were discontinued, the fentanyl self-administration baseline was re-established, and effects of naltrexone were determined a second time. Thereafter, $0.1 \mathrm{mg} / \mathrm{kg}$ MCAM was studied in the same manner as the previous test. Cocaine was not tested in one monkey after this dose of MCAM because responding for fentanyl returned to baseline within 3 days of the injection. For the other monkeys, cocaine was substituted every fourth session, as before, with at least 7 sessions (range: 7-15) conducted after MCAM administration, at which point cocaine sessions were discontinued, the fentanyl self-administration baseline was re-established, and the naltrexone dose-effect curve was determined a third time.

Effects of repeated MCAM administration

Effects of repeated MCAM administration were tested following the third naltrexone dose-effect curve determination. A dose of $0.32 \mathrm{mg} / \mathrm{kg}$ MCAM was given $60 \mathrm{~min}$ prior to a fentanyl selfadministration session and testing commenced, as before, with cocaine being substituted for fentanyl every fourth session. Four additional injections of $0.32 \mathrm{mg} / \mathrm{kg}$ MCAM were given 12 days apart (i.e., 12, 24, 36, and 48 days, respectively, after the initial MCAM injection). Fentanyl and cocaine sessions continued to alternate after the fifth MCAM injection for at least 38 (range: 38-66) additional sessions.
Plasma collection and analysis

Blood was collected in 2 groups of monkey ( $n=4$ per group) for analysis of MCAM concentrations in plasma. In one group of monkeys ( 4 males), $0.32 \mathrm{mg} / \mathrm{kg}$ MCAM was administered s.c. on one occasion; blood was collected by acute puncture of the saphenous vein before and 15, 30, 45, 60, 120, and $360 \mathrm{~min}$ as well as 24 and $48 \mathrm{~h}$ after the injection. The other group comprised the four monkeys in the self-administration experiment that recieved repeated MCAM injections. Blood was collected immediately following the self-administration session on the day in which MCAM was given (Day 0 ) and on each of the following two days (Days 1 and 2); thus, sampling times were approximately $150 \mathrm{~min}$ (60 min pretreatment plus $90 \mathrm{~min}$ session), 1 day, and 2 days following each administration of $0.32 \mathrm{mg} / \mathrm{kg}$ MCAM. Samples were put in 3-ml EDTA tubes (Vacutainer; Becton, Dickson and Company, Franklin Lakes, NJ, USA) then immediately placed on ice and shielded from light. The tubes were centrifuged at $3000 \mathrm{~g}$ for $10 \mathrm{~min}$ at $15^{\circ} \mathrm{C}$; plasma was collected in polypropylene tubes and stored at $-80^{\circ} \mathrm{C}$.

\section{HPLC/MS/MS system and analytical parameters}

The high-pressure liquid chromatography (HPLC) system consisted of a Shimadzu CBM-20A controller (Shimadzu Scientific Instruments, Columbia, MD, USA), an LC-20AD pump with a FCV-20AL mixing chamber, a SIL-20A/HT autosampler, and an AB Sciex API 3200 tandem mass spectrometer with turbo ion spray (AB Sciex, Framingham, MA, USA). The analytical column was an Ace Excel 3 Super $\mathrm{C} 18(3 \times 75 \mathrm{~mm}, 3 \mu)$ purchased from MacMod (Chadds Ford, PA, USA) and maintained at $26^{\circ} \mathrm{C}$ during the chromatographic runs in a Shimadzu CTO-20A column oven. Mobile phase A was $0.1 \%$ formic acid dissolved in Milli-Q water, and mobile phase B was $0.1 \%$ formic acid dissolved in acetonitrile. The flow rate of the mobile phase was $0.4 \mathrm{ml} / \mathrm{min}$. The gradient was 0-1 min, 20\% B; 1.1-6 min, 20-99\% B (linear); 6.1-8 min, 99\% B; 8.1-10 min, 99-20\% B (linear). The Q1/Q3 transition used to quantify MCAM was $485.5-145.3 \mathrm{~m} / \mathrm{z}$.

Milli-Q water was used for preparation of all solutions (Millipore, Billerica, MA, USA). MCAM super-stock solutions were prepared in methanol at a concentration of $1 \mathrm{mg} / \mathrm{ml}$ and stored in aliquots at $-80^{\circ} \mathrm{C}$. Working stock solutions $(1$ or $10 \mu \mathrm{g} / \mathrm{ml})$ of MCAM were prepared fresh each day from the super-stock solutions and used to spike the calibrator samples. To determine plasma concentrations of MCAM, $200 \mu \mathrm{l}$ of calibrator and unknown plasma samples were mixed with $2 \mathrm{ml}$ of a mobile phase B $(0.1 \%$ formic acid dissolved in acetonitrile). The samples were vortexed for $2 \mathrm{~min}$, shaken for $30 \mathrm{~min}$, and then centrifuged at $3200 \mathrm{~g}$ for $30 \mathrm{~min}$ at $23^{\circ} \mathrm{C}$. Supernatants were dried to residue under a stream of nitrogen and residues were dissolved in $100 \mu$ of $0.1 \%$ formic acid in water/acetonitrile (1:1). Then, $10 \mu \mathrm{l}$ of the final samples were injected into the liquid chromatography-tandem mass spectrometry system. The peak areas of MCAM were compared to a linear regression of peak areas of spiked MCAM calibrator samples containing concentrations of $0,1,5,10,50,100,500$, and $1000 \mathrm{ng} / \mathrm{ml}$ of MCAM to calculate MCAM concentrations in unknown samples. The plasma concentrations of MCAM were expressed as $\mathrm{ng} / \mathrm{ml}$.

\section{Data analyses}

The primary dependent measure for the self-administration experiments was the number of infusions obtained. Changes in body weight were also quantified by taking the difference between the weight each day and the weight immediately preceding an MCAM injection. Effects of dose, day, and determination (naltrexone only) as well as the interaction between factors were analyzed using a one- or two-way, repeated measures analysis of variance (ANOVA). The potency of naltrexone was quantified by estimating the $\mathrm{ED}_{50}$ using linear interpolation; changes in the potency of naltrexone were analyzed using log 
$\mathrm{ED}_{50}$ values and a one-way, repeated measures ANOVA. Plasma MCAM concentrations were plotted for individual monkeys as a function of time since MCAM administration. Half-life $\left(T_{1 / 2}\right)$ was estimated by calculating half of the maximum concentration observed in plasma $\left(C_{\max }\right)$ and then interpolating between the two data points immediately surrounding that concentration. Half-life was the difference between the time obtained by interpolation and the observed time to reach the maximum concentration $\left(T_{\max }\right)$. Effects of day and injection number on plasma MCAM concentrations during repeated administration were analyzed using a two-way, repeated-measures ANOVA. Post hoc analyses were conducted using Dunnett's test [20]. Statistical analyses were conducted using NCSS, version 10.0.13 (Kaysville, UT, USA).

\section{Drugs}

The National Institute on Drug Abuse Drug Supply Program (Rockville, MD, USA) generously provided fentanyl hydrochloride, cocaine hydrochloride, and naltrexone hydrochloride. Methocinnamox (MCAM) was synthesized [8] and purchased (Syncom, Groningen, NL). Fentanyl, cocaine, and naltrexone were dissolved in $0.9 \%$ sterile saline, whereas MCAM was dissolved in $10 \% \mathrm{w} / \mathrm{v}$ 2 -hydroxypropyl- $\beta$-cyclodextrin. Fentanyl and cocaine were injected i.v. during self-administration sessions in a volume of $1 \mathrm{ml}$ per $10 \mathrm{~kg}$ of body weight; naltrexone and MCAM were injected s.c. in a volume of approximately $0.32 \mathrm{ml}$ per $10 \mathrm{~kg}$ of body weight. For pharmacokinetic studies, all reagents were purchased either from Sigma-Aldrich (St. Louis, MO, USA) or Fisher (Suwanee, GA, USA) and were HPLC grade or higher. Methocinnamox (MCAM) analytical standard was purchased from Syncom.

\section{RESULTS}

Effects of acute naltrexone administration

Monkeys obtained, on average, between 24.5 and 26.0 infusions of $0.00032 \mathrm{mg} / \mathrm{kg} /$ infusion fentanyl during baseline sessions and following saline pretreatment (data above "Baseline" and "S", Fig. 1a, b). During the first determination, naltrexone dosedependently decreased responding for fentanyl, with 0.01 and $0.032 \mathrm{mg} / \mathrm{kg}$ significantly decreasing the number of infusions on the day of treatment compared to baseline; responding returned to baseline levels the following day (Fig. 1a). A two-way ANOVA indicated a significant main effect of dose $(F[2,35]=13.4, p<$ $0.001)$ and day $(F[2,35]=120.4, p<0.0001)$ as well as a significant dose-by-day interaction $(F[4,35]=21.1, p<0.0001)$. When tested later in the study, naltrexone again dose-dependently decreased responding for fentanyl (Fig. 1b), with effects lasting less than one day (data not shown). A two-way ANOVA on naltrexone dose-effect data indicated a main effect of dose of naltrexone $(F[3,39]=51.7, p<0.0001)$, but no main effect of determination or dose-by-determination interaction. Mean $(95 \% \mathrm{Cl}) \mathrm{ED}_{50}$ values for naltrexone were 0.006 (0.005-0.007), 0.007 (0.004-0.013), and $0.006(0.004-0.009) \mathrm{mg} / \mathrm{kg}$ for the first, second, and third determinations, respectively; according to a one-way ANOVA, $\mathrm{ED}_{50}$ values did not differ significantly across determinations.

\section{Effects of acute MCAM administration}

Before tests with MCAM, monkeys obtained, on average, between 21.5 and 23.3 infusions of fentanyl and between 20.3 and 23.3 infusions of cocaine during baseline sessions (data above " $B$ ", Fig. 2a-c). When given acutely, MCAM decreased responding for fentanyl but not cocaine (squares and circles, respectively, Fig. 2a, b). Following a single administration of $0.1 \mathrm{mg} / \mathrm{kg}$ of MCAM, there was a significant main effect of day on the number of fentanyl $(F[6,27]=11.6, p<0.0001)$, but not cocaine, infusions, with the number of fentanyl infusions being significantly lower than baseline for up to 2 days after MCAM administration (filled symbols, Fig. 2a). Following a single administration of $0.32 \mathrm{mg} / \mathrm{kg}$
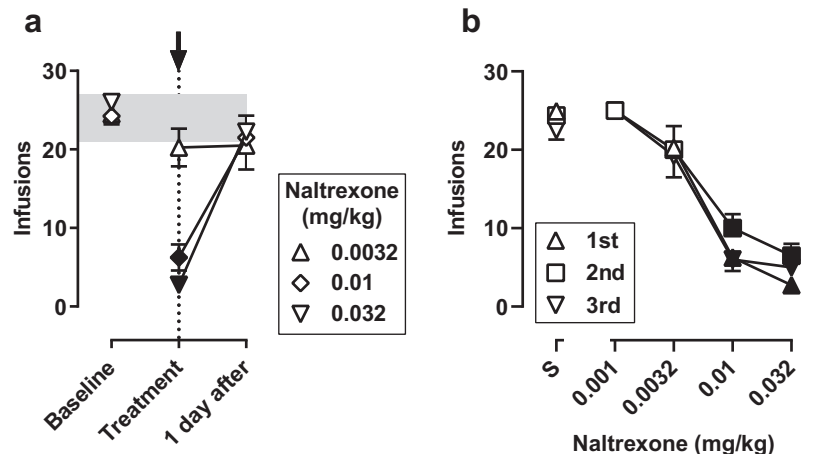

Fig. 1 Time- and dose-dependent effects of naltrexone on fentanyl self-administration. a Time course of effects of naltrexone on fentanyl self-administration $(n=4)$. The number of fentanyl infusions per session is plotted across three consecutive sessions: immediately preceding a test ("Baseline"), 15 min after an injection of naltrexone ("Treatment"), and 1 day after an injection of naltrexone ("1 day after"). Each symbol indicates a test with a different dose of naltrexone. The shaded region shows the range across baseline sessions for individual monkeys, and the arrow and vertical dashed line indicate the day on which naltrexone was administered. b Naltrexone dose-effect curves plotted for each of three determinations during the course of the study $(n=4$, except as indicated below). The mean number of fentanyl infusions is plotted for pretreatment with saline (above S) and increasing doses of naltrexone. All monkeys were tested with $0.0032,0.01$, and $0.032 \mathrm{mg} / \mathrm{kg}$ for the first determination. The range of doses tested varied across monkeys for the second and third determinations depending on effects of naltrexone, as follows: second determination, $0.001 \mathrm{mg} / \mathrm{kg}(n=1)$ and $0.032 \mathrm{mg} / \mathrm{kg}(n=2)$; third determination, $0.001 \mathrm{mg} / \mathrm{kg}(n=1)$ and $0.032 \mathrm{mg} / \mathrm{kg}(n=1)$. For both panels, symbols represent the mean $( \pm 1$ SEM); filled symbols indicate data that are significantly different from baseline (a) or saline (b) according to a Dunnett's test $(p<0.05)$.

MCAM, there was a significant main effect of day on the number of fentanyl $(F[24,98]=6.32, p<0.0001)$, but not cocaine, infusions, with the number of fentanyl infusions being significantly lower than baseline for up to 13 days after MCAM administration (Fig. 2b). A two-way ANOVA on fentanyl infusions, including the treatment day as well as the first 6 days after MCAM administration, indicated there was a main effect of dose of MCAM $(F[1,55]=$ $64.1, p<0.0001)$ and day $(F[6,55]=12.3, p<0.0001)$ as well as a dose-by-day interaction $(F[6,55]=7.2, p<0.0001)$. In contrast, for cocaine infusions, there was no main effect of dose of MCAM, no main effect of day, and no dose-by-day interaction.

Effects of repeated MCAM administration

Repeated treatment with $0.32 \mathrm{mg} / \mathrm{kg}$ MCAM also decreased responding for fentanyl, but not cocaine, with effects lasting for the duration of treatment (Fig. 2c). There was a significant main effect of day on fentanyl $(F[72,291]=3.85, p<0.0001)$, but not cocaine, infusions, with the number of fentanyl infusions remaining significantly lower than baseline consistently for up to 72 days during the course of repeated treatment. The number of fentanyl infusions returned to baseline levels at a slightly lower rate following repeated MCAM treatment, with infusions remaining significantly lower than baseline for up to 21 days following repeated administration and for up to 13 days following acute administration (compare the recovery of responding for fentanyl in Fig. 2b with data from Fig. $2 c$ beginning with the last MCAM injection). A two-way ANOVA indicated a main effect of day $(F[33,270]=13.1, p<0.0001)$, a main effect of treatment condition $(F[1,270]=7.9, p=0.005)$, as well as a day-by-treatment interaction $(F[33,270]=1.6, p=0.03)$.

During repeated MCAM treatment, there was a modest but significant effect of day on body weight $(F[96,387]=1.62$, 

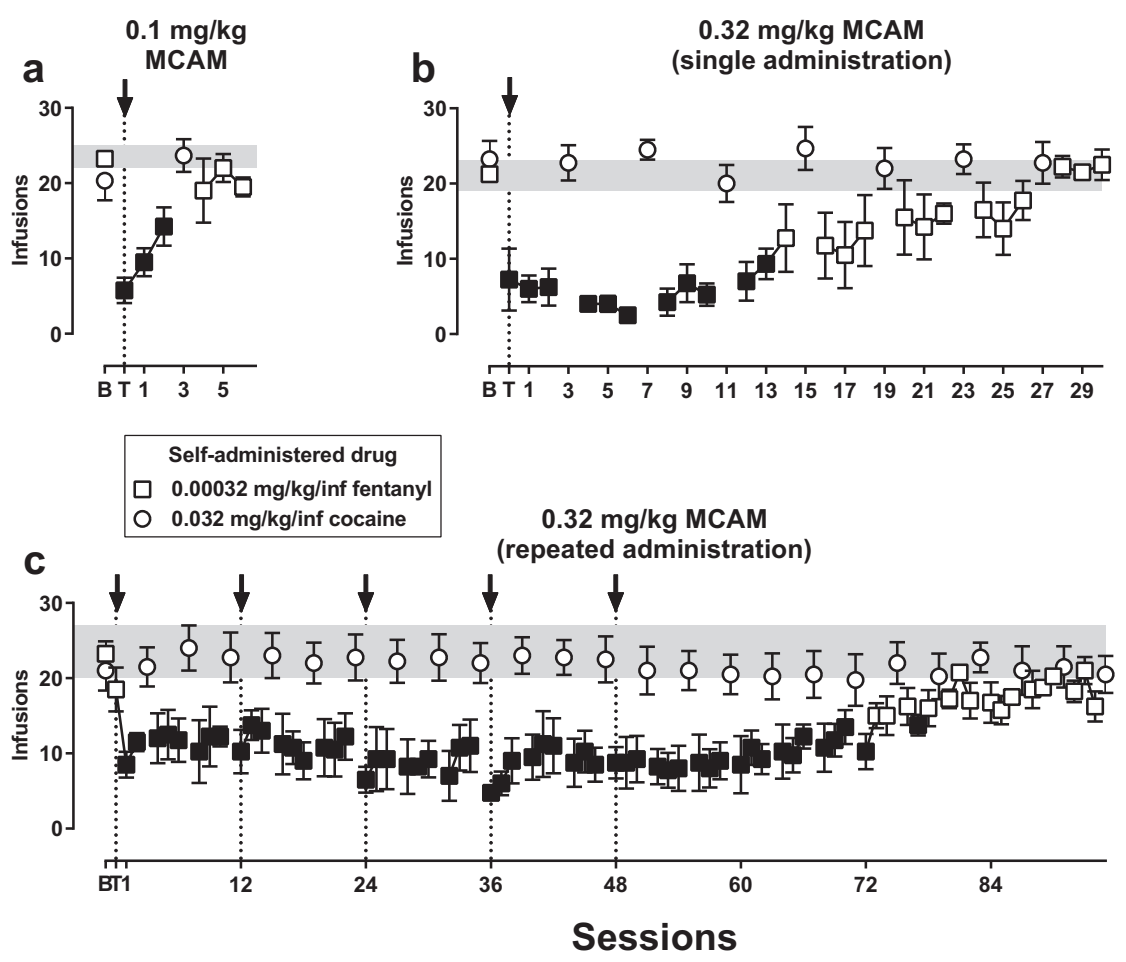

Fig. 2 Effects of acute or repeated treatment with MCAM on self-administration of fentanyl or cocaine. Panels a and $\mathbf{b}$ show effects of a single injection of 0.1 and $0.32 \mathrm{mg} / \mathrm{kg}$ MCAM, respectively; panel c shows effects of repeated injections of $0.32 \mathrm{mg} / \mathrm{kg}$ MCAM, with injections separated by 12 days. For all panels, $n=4$ except for cocaine data in panel a which is $n=3$ (see "Effects of acute MCAM administration" for more information). The number of infusions is plotted across consecutive days; symbols above "B" indicate data from the sessions preceding MCAM injection, whereas symbols above " $\mathrm{T}$ " indicate data from the session on the day of treatment (i.e., 60 min after MCAM injection) for panels $\mathbf{a}$ and $\mathbf{b}$ or the first MCAM injection in panel $\mathbf{c}$. Symbols represent the mean ( $\pm 1 \mathrm{SEM})$; filled symbols indicate data that are significantly different from baseline according to a Dunnett's test $(p<0.05)$. The shaded region shows the range of the baseline sessions across individual monkeys, and the arrows and vertical dashed lines indicate the days on which MCAM was administered.

$p=0.001$; data not shown). Average gain in body weight was approximately $0.5 \mathrm{~kg}$ by the end of the experiment (Day 95); one monkey accounted for the increase almost entirely. There was no evidence of marked weight loss $(\geq 0.5 \mathrm{~kg})$ in any monkey following MCAM administration.

MCAM plasma levels

Following a single injection of $0.32 \mathrm{mg} / \mathrm{kg}$ MCAM s.c., plasma levels in individual monkeys reached peak concentrations, which ranged from 10.6 to $26.0 \mathrm{ng} / \mathrm{ml}, 15-45 \mathrm{~min}$ after the injection (Fig. 3). These concentrations decreased with a half-life ranging from 13.7 to $199.8 \mathrm{~min}$. In the self-administration group, mean MCAM plasma concentration ranged from, on average, $4.78-10.32 \mathrm{ng} / \mathrm{ml}$ immediately following the session (i.e., $150 \mathrm{~min}$ after administration) on the day $0.32 \mathrm{mg} / \mathrm{kg}$ MCAM was administered and was much lower the following days, ranging from 0.93 to $1.97 \mathrm{ng} / \mathrm{ml} 1$ day after injection and from 0.73 to $1.78 \mathrm{ng} / \mathrm{ml}$ two days after injection (Fig. 4). There was a main effect of day $(F[3,61]=5.5, p=0.003)$ on plasma concentrations, but there was no main effect of test and no day-bytest interaction. MCAM plasma levels on the day of injection (days 0 , $12,24,36$, and 48) were significantly higher than control. One value for one monkey was excluded from the analysis (day 36, immediately following the fourth MCAM injection; Fig. 4) because that value $(27.7 \mathrm{ng} / \mathrm{ml})$ was more than 6 standard deviations higher than the mean plasma levels measured across the other four injections in the same monkey, which were $5.9,5.2,8.1$, and $6.8 \mathrm{ng} / \mathrm{ml}$.

\section{DISCUSSION}

Opioid abuse and overdose continue to be significant public health challenges despite the availability of effective medications,

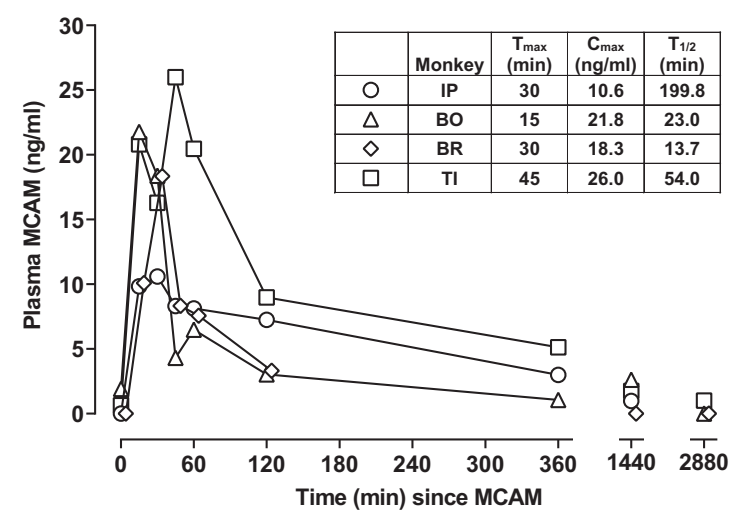

Fig. 3 Plasma concentration of MCAM following acute MCAM administration. Plasma MCAM concentration in $\mathrm{ng} / \mathrm{ml}$ is plotted as a function of time since MCAM administration. Symbols show data from indivdiual monkeys. The table inset shows pharmacokinetic parameters derived for each monkey. $T_{\max }$ is the observed time to reach the maximum concentration; $C_{\max }$ is the maximum concentration observed in plasma; and $T_{1 / 2}$ is an estimate of the time needed to reduce plasma levels of MCAM to half of the maximum. One monkey was excluded from the $360 \mathrm{~min}$ time point and another from the 2880 min time point because samples were not available for analysis.

including methadone, buprenorphine, naltrexone, and naloxone. MCAM is a pseudoirreversible $m u$ opioid receptor selective antagonist [8-10] that blocks the reinforcing and ventilatorydepressant effects of opioids in nonhuman primates, with effects of a single injection lasting days to weeks without any evidence of 


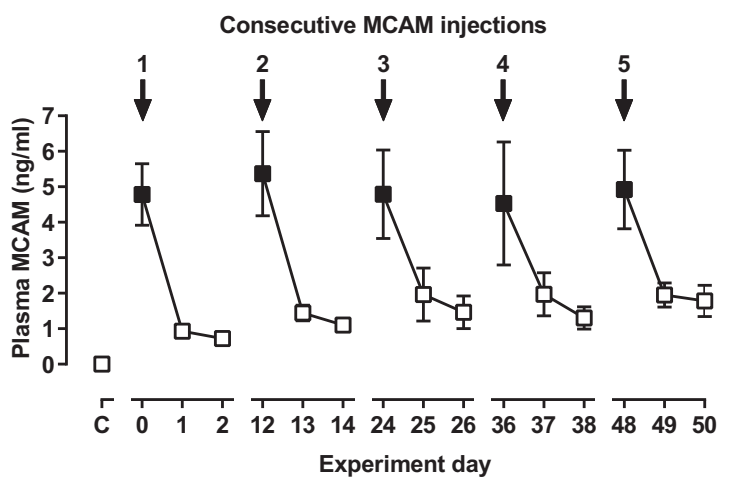

Fig. 4 Plasma concentration of MCAM following repeated MCAM administration. Plasma was collected immediately following the self-administration session on the day in which MCAM was given and on each of the following two days. Each triad of data points shows plasma MCAM concentration in $\mathrm{ng} / \mathrm{ml}$ for an MCAM injection. Numbers along the abscissa indicate days since the first MCAM injection of the test; the data point above " $C$ " indicates concentration in plasma collected before the experiment and prior to the first MCAM administration. Each data point represents the mean $( \pm 1$ SEM) for four monkeys, except for day 25 , when blood was not collected from one monkey, and for day 36 , when an outlier was excluded (see "Results" for details). Filled symbols indicate means that are statistically different from baseline (data point above " ${ }^{\prime \prime}$ ) according to a Dunnett's test $(p<0.05)$.

adverse effects $[11,12]$. Previous studies have demonstrated that MCAM selectively reduces opioid self-administration, although it is important to know whether effects of MCAM are sustained over extended treatment and whether extended treatment with MCAM results in long-term changes that might have important consequences. This study evaluated MCAM for its capacity to reduce self-administration of the potent and high efficacy mu opioid receptor agonist fentanyl, characterized the effectiveness of repeated treatment with MCAM, and related behavioral effects of MCAM to its pharmacokinetic properties. Results of this study showed the following: (1) MCAM selectively decreased selfadministration of fentanyl but not cocaine; (2) effects of a single injection of MCAM on fentanyl self-administration lasted much longer than those of naltrexone; (3) MCAM remained effective for over 2 months during a course of repeated, intermittent treatment; (4) recovery of responding for fentanyl after discontinuation of repeated MCAM administration was not markedly different from recovery following a single MCAM injection; and (5) MCAM remained effective despite very low concentrations in plasma.

Effects of acute MCAM administration

When given acutely, MCAM and naltrexone decreased responding for fentanyl at doses that also decreased responding for heroin and reduced choice of remifentanil over food in rhesus monkeys [12]. Effects of naltrexone were relatively short-lived as responding returned to baseline levels the next day. In contrast, effects of a single injection of MCAM lasted much longer than those of naltrexone with the larger dose of MCAM decreasing fentanyl selfadministration for up to 2 weeks. This duration of action is comparable to a previous study in which $0.32 \mathrm{mg} / \mathrm{kg} \mathrm{MCAM}$ decreased heroin self-administration in rhesus monkeys for an average of 10 days following a single injection [12]. In comparison, the same dose of MCAM blocked the ventilatory-depressant effects of very large doses of heroin, shifting the dose-effect curve rightward at least 10 fold, for at least 4 days, with the heroin dose-effect curve gradually returning to control over the course of 8 days [11].

Only one dose of fentanyl was tested in the current study. Given that the self-administration dose-effect curves are usually biphasic (i.e., "inverted-U" shaped), decreased responding for a single dose could indicate a leftward or rightward shift in the dose-effect curve, with a leftward shift indicating enhancement of reinforcing effects and a rightward shift indicating attenuation. Based solely on the current data, it is not possible to determine whether MCAM enhanced or attenuated the reinforcing effects of fentanyl. However, in previous studies with rhesus monkeys, MCAM shifted dose-effect curves for choice of remifentanil over food [12] and the ventilatory-depressant effects of heroin [11] rightward in a dose-dependent fashion; thus, it appears likely that decreased self-administration of fentanyl in the current study reflected attenuation, and not enhancement, of the reinforcing effects of fentanyl. MCAM did not alter self-administration of cocaine when it was available intermittently in the same monkeys during the same experiment. Selective attenuation of opioid, but not cocaine, self-administration by an opioid receptor antagonist is consistent with previous studies [12, 21] and provides additional evidence that effects of MCAM on fentanyl self-administration were mediated through blockade of mu opioid receptors rather than through other mechanisms such as generalized suppression of operant behavior.

\section{Effects of repeated MCAM administration}

Because successful management of OUD requires long-term, and usually repeated, treatment, this study also evaluated effects of repeated MCAM administration. MCAM continuously reduced fentanyl self-administration during a treatment regimen in which MCAM was administered once every 12 days, with no evidence of a change in its effectiveness. The treatment interval was selected to use the longest inter-injection interval that would be predicted to produce a continuous reduction in self-administration of fentanyl, based on results obtained after acute dosing with MCAM. Although repeated bolus injections might not be the treatment regimen when using MCAM to treat OUD, these data indicate that it will remain effective over an extended period. Moreover, these results set the stage for developing other formulations (e.g., pills) that could be taken every week or two or formulations that deliver very small doses of MCAM over an extended period of time, which could markedly improve treatment compliance by reducing the frequency of dosing required and ensuring exposure to the medication for a very long time.

Like mu opioid receptor antagonists approved by the US Food and Drug Administration to treat OUD and opioid overdose (naltrexone and naloxone, respectively), MCAM would be devoid of many adverse effects associated with the agonists methadone and buprenorphine such as potential for abuse, ventilatory depression, and potentially adverse interactions with drugs that also decrease ventilation such as benzodiazepines and alcohol. Although MCAM has affinity for mu, kappa, and delta opioid receptors, it binds pseudoirreversibly only to mu opioid receptors [e.g., 8, 10]. Thus, long-term blockade of kappa and delta opioid receptors is not a concern with MCAM, further indicating that MCAM would be an improvement over extended-release formulations of naltrexone (i.e., Vivitrol), which would cause sustained blockade of all three types of opioid receptors. Moreover, MCAM did not decrease responding for food or alter heart rate, blood pressure, or body temperature up to a dose of $3.2 \mathrm{mg} / \mathrm{kg}$, tenfold larger than the dose that markedly decreases opioid selfadministration [12], suggesting a low risk for adverse effects. Although irreversible mu opioid receptor antagonists such as $\beta$-funaltrexamine can disrupt feeding behavior [e.g., 22], repeated treatment with MCAM at a frequency that continuously decreased fentanyl self-administration for more than 2 months did not result in significant weight loss or produce other overt signs of disrupted feeding or drinking in monkeys.

Long-term blockade of mu opioid receptors might perturb the endogenous opioid system, which could manifest as alterations in 
1992

effects of drugs acting at opioid receptors. Effects of naltrexone were determined between MCAM tests to probe for such effects, and the potency of naltrexone to decrease opioid selfadministration determined immediately before and following recovery of responding after MCAM treatment was not significantly different [current study; 12]. Naltrexone was not tested after repeated MCAM in the current experiment; however, recovery of responding for fentanyl could be considered a surrogate measure of the return of opioid system sensitivity $[23,24]$. Responding in all monkeys returned to baseline on generally the same timeline following acute and repeated treatment with MCAM, suggesting there was no marked acceleration or delay in opioid system normalization. The lengthy recovery in fentanyl self-administration following acute and repeated MCAM treatment suggests that patients would remain protected against abuse-related effects of opioids for days or even weeks following discontinuation of MCAM treatment. Moreover, given that the duration of protection against opioid-induced ventilatory depression parallels effects on opioid self-administration [11, 12], patients would also remain protected against opioid overdose.

MCAM pharmacodynamics and pharmacokinetics

The long duration of action of MCAM is thought to be due, at least in part, to irreversible binding to $m u$ opioid receptors. That is, MCAM binds to but does not dissociate from the receptor, rendering the receptor unavailable to other ligands; recovery of function is thus related to the restoration of functional receptors. At sufficient levels of receptor occupancy, irreversible binding of mu opioid receptors by MCAM would be expected to provide sustained and insurmountable protection against the reinforcing effects that contribute to abuse and the ventilatory-depressant effects that lead to overdose and death. However, whether an antagonist such as MCAM will provide insurmountable protection depends on several factors including the number of receptors occupied by the antagonist, the efficacy requirement of the $m u$ opioid receptor agonist-mediated effect, and the intrinsic efficacy of the agonist [e.g., 25]. Therefore, it is possible that, at some doses, MCAM will provide greater protection against adverse effects of opioids with moderate efficacy than against adverse effects of opioids with higher efficacy. Characterizing the scope and magnitude of the type of protection that MCAM provides is an ongoing effort. Nevertheless, studies to date indicate that MCAM provides substantial and long-lasting protection against the reinforcing and ventilatory-depressant effects of opioids with high efficacy such as heroin and fentanyl [current study, 11, 12].

To date, there is no direct evidence of pseudoirreversible binding of MCAM in vivo. It is possible that MCAM remains in circulation providing continuous blockade of $m u$ opioid receptors. Slow clearance and/or tissue sequestration might help to sustain high levels of circulating MCAM long after its administration. Such effects would be similar to those of repeated or continuous infusion of competitive, reversible antagonists such as naltrexone on opioid self-administration [e.g., 26]. In the current study, the pharmacokinetic profile of MCAM was examined to determine whether sustained plasma concentrations account for its long duration of action. Data appeared to represent biphasic elimination, although there were too few data points to discriminate between one- and two-phase models and variability was unexpectedly high when the data were fit to the simpler (one-phase) model. Nevertheless, results obtained in individual monkeys show that MCAM remained effective even when plasma concentrations were very low, supporting the view that pharmacodynamic factors (i.e., pseudoirreversible binding to $m u$ opioid receptors) play a significant role in its long-lasting effects, although, at this time a role for long-term sequestration in the central nervous system, as is the case with the long-acting kappa opioid receptor antagonist nor-binaltorphamine [27], cannot be ruled out. Moreover, plasma MCAM concentration obtained on the day of the injection did not differ systematically across repeated administrations suggesting that MCAM did not accumulate in the blood or alter its own pharmacokinetic profile when injections were separated by 12 days.

\section{CONCLUSIONS}

These results confirm and extend previous studies, demonstrating that MCAM selectively reduces opioid self-administration for a very long time after a single administration and remains effective with repeated administration. The long duration of action of MCAM is likely due, at least in part, to pseudoirreversible binding to $m u$ opioid receptors, suggesting it can insurmountably block the abuse- and overdose-related effects of opioids, which, in turn, would markedly reduce the risk of overdose and death. Such a long duration of antagonist action might be preferable for treating OUD in some patients for whom access to adequate health care is limited. Moreover, treatment compliance would likely increase in so far as binding of MCAM to mu opioid receptors cannot easily be reversed, for example, by removing an implant. Taken together with previous studies, these data indicate that MCAM could be an effective, long-acting treatment for OUD and overdose which would be a desperately needed addition to the armamentarium currently available to combat the opioid crisis.

\section{FUNDING AND DISCLOSURE}

This work was supported by R01DA005018 (CPF), R01DA048417 (CPF), and R01DA007315 (SMH) from the National Institutes of Health, grant AQ-0039 from the Welch Foundation (CPF), and the Nancy U. Karren Endowment in Psychiatry (MAJ).

The content is solely the responsibility of the authors and does not necessarily represent the official views of the National Institutes of Health. CPF is co-holder of a pending US patent on methocinnamox; the remaining authors declare no competing interests.

\section{ACKNOWLEDGEMENTS}

The authors thank Matthew Deande, Candace Earlywine, Jade Juarez, Anastassia Nelson, and Samuel Womack for excellent technical assistance.

\section{AUTHOR CONTRIBUTIONS}

DRM, LRG, and CPF participated in the research design; DRM, LRG, and JJS conducted the experiments; AD and SMH contributed new reagents; DRM, LRG, JJS, and MAJ performed data analysis; DRM, LRG, JJS, MAJ, AD, SMH, and CPF wrote or contributed to the writing of the paper.

Publisher's note Springer Nature remains neutral with regard to jurisdictional claims in published maps and institutional affiliations.

\section{REFERENCES}

1. Hedegaard H, Bastian BA, Trinidad JP, Spencer M, Warner M. Drugs most frequently involved in drug overdose deaths: United States, 2011-2016. Natl Vital Stat Rep. 2018;67:1-14. https://www.cdc.gov/nchs/data/nvsr/nvsr67/nvsr67_09508.pdf Accessed 30 December 2019.

2. Jones CM, Paulozzi LJ, Mack KA. Centers for Disease Control and Prevention (CDC) Alcohol involvement in opioid pain reliever and benzodiazepine drug abuse-related emergency department visits and drug-related deaths-United States, 2010. MMWR Morb Mortal Wkly Rep. 2014;63:881-5. https://www.ncbi. nlm.nih.gov/pmc/articles/PMC4584609/ Accessed 30 December 2019.

3. Jones CM, McAninch JK. Emergency department visits and overdose deaths from combined use of opioids and benzodiazepines. Am J Prev Med. 2015;49:493-501.

4. Nunes EV, Bisaga A, Krupitsky E, Nangia N, Silverman BL, Akerman SC, et al. Opioid used and dropout from extended-release naltrexone in a controlled trial: implications for mechanism. Addiction. 2019;115:239-46.

5. Wakeman SE, Larochelle MR, Ameli O, Chaisson CE, McPheeters JT, Crown WH, et al. Comparative effectiveness of different treatment pathways for opioid use disorder. JAMA Netw Open. 2020;3:e1920622. 
Effects of acute and repeated treatment with methocinnamox, a mu opioid... DR Maguire et al.

6. Volkow ND, Jones EB, Einstein EB, Wargo EM. Prevention and treatment of opioid misuse and addiction: a review. JAMA Psychiatry. 2019;76:208-16.

7. Portoghese PS, Larson DL, Sayre LM, Fries DS, Takemori AE. A novel opioid receptor site directed alkylating agent with irreversible narcotic antagonistic and reversible agonistic activities. J Med Chem. 1980;23:233-4.

8. Broadbear JH, Sumpter TL, Burke TF, Husbands SM, Lewis JW, Woods JH, et al. Methocinnamox is a potent, long-lasting, and selective antagonist of morphinemediated antinociception in the mouse: comparison with clocinnamox, $\beta$ funaltrexamine, and $\beta$-chlornaltrexamine. J Pharm Exp Ther. 2000;294:933-40.

9. Peckham EM, Barkley LM, Divin MF, Cicero TJ, Traynor JR. Comparison of the antinociceptive effect of acute morphine in female and male Sprague-Dawley rats using the long-lasting mu-antagonist methocinnamox. Brain Res. 2005;1058:137-47.

10. Gerak LR, Minervini V, Latham E, Ghodrati S, Lillis K, Wooden J, et al. Methocinnamox produces long-lasting antagonism of the behavioral effects of $\mu$-opioid receptor agonists but not prolonged precipitated withdrawal in rats. J Pharm Exp Ther. 2019;371:507-16.

11. Gerak LR, Maguire DR, Woods JH, Husbands SM, Disney A, France CP. Reversal and prevention of the respiratory-depressant effects of heroin by the novel $\mu$-opioid receptor antagonist methocinnamox in rhesus monkeys. J Pharm Exp Ther. 2019;368:229-36.

12. Maguire DR, Gerak LR, Woods JH, Husbands SM, Disney A, France CP. Longlasting effects of methocinnamox on opioid self-administration in rhesus monkeys. J Pharm Exp Ther. 2019;368:88-99.

13. Spencer MR, Warner M, Bastian BA, Trinidad JP, Hedegaard H. Drug overdose deaths involving fentanyl, 2011-2016. Natl Vital- Stat Rep. 2019;68:1-19. https:// www.cdc.gov/nchs/data/nvsr/nvsr68/nvsr68_03-508.pdf Accessed 30 December 2019.

14. Mello NK, Negus SS. Preclinical evaluation of pharmacotherapies for treatment of cocaine and opioid abuse using drug self-administration procedures. Neuropsychopharmacology. 1996;14:375-424.

15. Maguire DR, Gerak LR, Cami-Kobeci G, Husbands SM, France CP, Belli B, et al OREX-1019: a novel treatment for opioid use disorder and relapse prevention. J Pharm Exp Ther. 2020;372:205-15.
16. Broadbear $\mathrm{JH}$, Winger $\mathrm{G}$, Woods $\mathrm{JH}$. Self-administration of fentanyl, cocaine and ketamine: effects on the pituitary-adrenal axis in rhesus monkeys. Psychopharmacology. 2004;176:398-406.

17. Ko MC, Terner J, Hursh S, Woods JH, Winger G. Relative reinforcing effects of three opioids with different durations of action. J Pharm Exp Ther. 2002;301:698-704.

18. Gerak LR, Galici R, France CP. Self administration of heroin and cocaine in morphine-dependent and morphine-withdrawn rhesus monkeys. Psychopharmacology. 2009;204:403-11.

19. Collins GT, Gerak LR, Javors MA, France CP. Lorcaserin reduces the discriminative stimulus and reinforcing effects of cocaine in rhesus monkeys. J Pharm Exp Ther. 2016:356:85-95.

20. Dunnett CW. A multiple comparison procedure for comparing several treatments with a control. J Am Stat Assoc. 1955;50:1096-121.

21. Winger G, Skjoldager P, Woods JH. Effects of buprenorphine and other opioid agonists and antagonists on alfentanil-and cocaine-reinforced responding in rhesus monkeys. J Pharm Exp Ther. 1992;261:311-7.

22. Ukai $M$, Holtzman SG. Effects of $\beta$-funaitrexamine on ingestive behaviors in the rat. Eur J Pharm. 1988;153:161-5.

23. Martin TJ, Dworkin SI, Smith JE. Alkylation of mu opioid receptors by betafunaltrexamine in vivo: comparison of the effects on in situ binding and heroin self-administration in rats. J Pharm Exp Ther. 1995;272:1135-40.

24. Zernig G, Lewis LW, Woods JH. Clocinnamox inhibits the intravenous selfadministration of opioid agonists in rhesus monkeys: comparison with effects on opioid agonist-mediated antinociception. Psychopharmacology. 1997;129:233-42.

25. Adams JU, Paronis CA, Holtzman SG. Assessment of relative intrinsic activity of mu-opioid analgesics in vivo by using beta-funaltrexamine. J Pharm Exp Ther 1990;255:1027-32.

26. Townsend EA, Negus SS, Poklis JL, Banks ML. Lorcaserin maintenance fails to attenuate heroin vs. food choice in rhesus monkeys. Drug Alcohol Depend. 2020;208:107848.

27. Kishioka S, Kiguchi N, Kobayashi Y, Yamamoto C, Saika F, Wakida N, et al Pharmacokinetic evidence for the long-lasting effect of nor-binaltorphimine, a potent kappa opioid receptor antagonist, in mice. Neurosci Lett. 2013;552:98-102. 